öffnen können, da diese dem collegialischen Sinne natürlich nicht förderlich, sondern schädlich sind.

D. Red.

\title{
4) Mittheilungen über den Zustand der Pharmacie und die Verhältnisse der Apotheker in Croatien.
}

(Aus einem Briefe des Apoth. Bérnoth in Warasdin an Dr. Meurer in Dresden.)

Croatien, Dalmatien und Slavonien bilden zusammen ein Königreich, welches unter dem Bannus von Croatien, der in Agram seinen Sitz hat, steht. Dies Königreich ist der ungarischen Krone untergeordnet und steht mit dieser unter dem Kaiser von Oesterreich, der zugleich König von Ungarn ist. Eine Universität ist in Croatien nicht, wohl aber einige Lyceen und Gymnasien; die Studien, so wie die Staatsprüfung müssen daher die Apotheker in Pesth oder Wien machen. Gremien der Apotheker existiren noch nicht, doch hat nan sich in Ungarn bemüht dieselben einzuführen. Die Medicinalverordnungen erhalten die Croatier von der ungarischen Statthalterei oder von der Universităt zu Pesth, doch ist im Allgemeinen die Medicinalpolizei gut: auch die Taxe wird von dort ausgegeben, doch ist die felate vom October 1843. Als Dispensatoriun gilt das ôsterreichische, wovon die letzte Ausgabe von 1836, doch soll bald ein ganz neues erscheinen. - Die Apotheken werden alle drei Jahre von den Stadtund Comitats-Physicis, von zwei Herren von der politischen Behörde unter Zuziehung eines Apothekers revidirt. Nach dem Gesetze sollen nur an Orten, wo wenigstens 6000 Einwohner sind, Apotheken angelegt werden.

Jeder, der als Apotheker lernen will, muss dem Physicus vorgestellt werden, der über seine geistigen und körperlichen Fähigkeiten zu entscheiden hat, ferner muss er diesem ein Sittenzeugniss und dass er wenigstens die vierte lateinische Classe absolvirt, vorlegen. Der Lehrherr muss über den sittlichen Wandel des Lehrlings wachen, denselben gut behandeln, ihn in den pharmaceutischen Manipulationen unterrichten und ihm Gelegenheit und Musse verschaffen, die Naturgeschichte, Botanik und Chemie zu erlernen, damit er die in der Pharmakopöe enthaltenen Arzneibereitungen kunstgerecht darstellen lerne; über alles diess muss der Lehrherr den Lehrling fleissig prüfen.

Nach einer vierjährigen Lehrzeit wird der Lehrling vom Physicus und einem Apotheker sowohl praklisch als theoretisch geprift, und nach gutem Bestehen demselben ein Lehrbrief von dem Examinator und dein Lehrherrn ausgefertigt.

Wer der Staatsprüfung sich unterwerfen will, muss 1) ein Zeugniss beibringen, dass er die oben beschriebene Lehrzeit gut überstanden, 2) belegen, dass er wenigstens zwei Jahre in einer ordentlichen Apotheke nit Lob und Zufriedenheit conditionirt, 3) muss er einen Taufschein vorzeigen, weil kein Jude Apotheker sein darf, 4) nuss er beweisen, dass er zwei Jahre auf einer Universität, im ersten specielle Naturgeschichte, Botanik, Waarenkunde, im zweiten Physik und Chemie studirt habe. Hat er diesem allem genügt, so kann er sich beim Decan der medicinischen Facultät melden, der ihn an den Professor der Chemie weist, unter dessen Aufsicht er zwei chemische Präparate zu fertigen hat; bringt der Candidat hierüber ein günstiges 
Zeugniss, so wird er zur mündlichen Prüfung zugelassen. Diese findet unter Vorsitz des Decans und vor dem Professor der Naturwissenschaften statt, wobei auch vorgelegte Pflanzen und Droguen bestimmt werden müssen. Die Prüfung soll ziemlich streng sein, und der Candidat erhält nach seinen Leistungen, entweder sufficienter, sat bene, bene, oder valde bene, als Censurgrad, und wird zum Magister der Pharmacie creirt. Für diese Statsprüfung sind 86 Gulden zu erlegen. Um nun eine Apotheke verwalten zu können, muss der Geprüfte noch einen Eid ablegen, der dem bei uns üblichen gleicht. - Hieraus ist zu ersehen und auch durch das Gesetz bestimmi, dass der Apotheker zu den Honoratioren gehört. (?) Die Apotheker sind in Croatien gänzlich militairfrei, sind auch frei von Einquartierung, sie stehen unter dem Staats - und Comitats - Physicus und unter dem Protomedicus der Provinz.

Will man irgendwo eine Apotheke errichten, so muss man zuerst bei der Localbehörde die Erlaubniss nachsuchen und erhalten, und sich dann von der kaiserl. Statthalterei die allerhöchste Erlaubniss nachsuchen, wo ihm dann ein Decret ausgefertigt wird. Jede einmal beslehende Apotheke kann ohne weitere Erlaubniss an cinen examinirten Apotheker verkauft werden. - In Croatien sind 27 Apotheken.

Hauptstadt: Agram. 1) Apotheke der barmherzigen Brüder.

2) Stadtapotheke, Joseph Zellinger, zum Schwarzen Adler.

3) Stadtapotheke, Georg Augustin, zum Salvator.

4) Hospitalapotheke, Ludwig Binder, zur Ungarischen Krone.

5) Bischöfliche Apotheke, Adolph Ladenh a u ffen, zum Ungarischen König.

6) Kaiserl. königl. Militairapotheke.

Carlsstadt. 7) Stadtapotheke, Alo is Meder, zum Adler.

Stadt Kourainitz.

8) Stadtapotheke, Johann Valehrich.

"Kreutz.

9) Stadtapotheke, Thikelonz, zum Adler.

"Warasdin.

10) Stadtapotheke, H einrich Bè r noth, Stellvertreter Ludwig Brotzky.

11) Stadtapotheke, Johann Halter, zum Mohren.

12) Kaiserl. königl. Militair - und Stadtapotheke, Heinrich Bèrnoth, zum Engel.

13) Comitatsapotheke, Theodor Todor, zum Salvator.

" Koapina. 14) Kaiseri. königl. Bergwerks - und Comitatsapotheke, Johann $Z$ ay.

"Klainetx.

"Jaska.

"Sovidsa.

"Petrina.

"Samabor.

St. Ivan.

"Glina.

Kloster Ivanic.

Stadt Bellovar.

" Kastanidsa.

15) Comitatsapotheke, Ferdinand Klerr.

16) Comitatsapotheke, Carl Odig.

17) Comitatsapotheke, Carl von Raphaclis.

18) Stadtapothelie, Panadz Tor.

19) Stadtapotheke, Nagg.

20) Comitatsapotheke, Joseph Ostodzky.

21) Stadtapotheke, Schickelang.

22) Franziskanerapotheke, J o s ep h Dok orich.

23) Kaiserl. königl. Militairapotheke.

24) Stadtapotheke, Vallen loerich.

25) Stadtapothelie, Franz Kubanyr. 
Stadt Sissek.

"Kutina.
26) Stadtapotheke, Carl Zajovitz.

27) Filialapotheke, Provisor Kussich.

(Aus einem Briefe vom Anfang d. J.) Den 1. Sept. 1845 werden sich die ungarischen Naturforscher in Fünfkirchen versammeln unter Vorsitz des Prof. Dr. Ehrmann aus Olmütz, des Dr. Wagner, Apotheker in Pesth, und unsers Mitgliedes, des Apothekers Bêrnoth in Warasdin, wo man sich bemühen will, einen Apothekerverein für Ungarn, Croatien, Slavonien und Dalmatien zu gründen. Man hofft hierdurch den in neuerer Zeit sehr zurückgekommenen Stand, in dem es dort besonders an guten Gehülfen fehlen soll, wieder zu beleben und zu heben.

\section{5) Die Versammlung der skandinavischen Natur- forscher in Christiania. Vom 12. bis zum 18. Juli 1844 .}

Christiania. Wenn die Naturforscherversammlungen In allen Ländern, wo sie bisher gehalten wurden, den Austausch von Ideen beförderten und zu wissenschaftlichen Leistungen anspornten, so findet diess ganz besonders seine Anwendung auf die Versammlung der skandinavischen Naturforscher. Norwegen, Schweden und Dänemark sind durch ihre geographische Lage und ihre verwandten Sprachen auf eine innige geistige Verbindung hingewiesen; dennoch hat eine solche Verbindung bisher nur unvollkommen statt gefunden, und es steht noch zu erwarten, dass sich dieselbe in dem Grade ausbilden werde, wie es in mehrfacher Beziehung wünschenswerth erscheint. Die Fortbildung der Wissenschaft verlangt, ausser der Protection der Regierung, eine Aufmunterung und Anerkennung, wie sie derselhen nur durch ein Publicum zu Theil werden kann, welches wissenschaftliche Leistungen zu würdigen versteht. In Ländern mit einer verhältnissmässig geringen Bevölkerung, deren Hauptinteressen, localer Umstände wegen, dem Handel, dem Ackerbau und der Schifffahrt zugewendet sind, kann ein solches Publicum nur sparsam verbreitet sein. Dänemark, Schweden und besonders Norwegen haben einen nicht zu läugnenden Mangel daran, und nur indem die drei verwandten Staaten sich die Hände reichen, kann dieser Mangel beseitigt werden. Manchen Schritt zu dieser Annäherung haben die Naturforschersammlungen bereits bewirkt (so z. B. wird nächstens eine für alle drei skandinavischen Staaten gemeinschaftliche medicinische Zeitschrift erscheinen), vieles bleibt aber in dieser Beziehung noch von denselben zil hoffen übrig. Erst wenn eine gemeinschaftliche Journal - Literatur in allen $Z$ weigen der Naturwissenschaften eine schnellere Circulation und allgemeinere Anerkennung der wissenschaftlichen Resultate bewirken wird, kann man erwarten, dass die Schranken fallen werden, welche bisher die drei verbrüderten skandinavischen Stämme verhindert haben, mit vereinigter Kraft als Ein Volk auf dem Gebiete der Wissenschaft aufzutreten.

Mit einer Liberalität, welche der Storthing trotz aller seiner Sparsamkeitsprincipien bisher stets an den Tag gelegt hat, wo es galt die Ehre der Nation und wissenschaftliche $Z$ wecke zu fördern, waren ron demselben bei seiner lelzten Sitzung 1800 Species (etwa 2700 Rıhlr. prouss. Courant) zur Bestreilung der nothwendigen offentlichen Aus- 